\title{
Efficacy of Dentin Bonding to Cervical Defects
}

\author{
Chihiro TANI, Kazuo ITOH, Hisashi HISAMITSU \\ and Sadao WAKUMOTO \\ Department of the Operative Dentistry \\ Showa University School of Dentistry \\ 2-1-1 Kitasenzoku, Ohta-ward \\ Tokyo 145-8515, Japan
}

Received July 15, 2001/Accepted September 26, 2001

\begin{abstract}
The bonding efficacy of sclerotic dentin was determined by measuring the polymerization contraction gap width of a commercial light-activated resin composite in a cervical defect and by measuring the micro-Vicker's hardness at the dentin adhesive surface; morphological characteristics were observed using a scanning electron microscope in extracted human incisors and premolars. Contraction gap formation was completely prevented when the cavity wall was primed with 35 vol\% glyceryl mono-methacrylate solution after $0.5 \mathrm{~mol} / \mathrm{L}$ EDTA conditioning. The contraction gap width was significantly decreased when the resin composite was filled into the sclerotic dentin cavity even when priming was omitted. These results suggest that the sclerotic dentin, which is frequently observed in cervical defects, should be preserved as a substrate because it exhibits an effect of dentin priming and is suitable for bonding.
\end{abstract}

Key words: Dentin bonding, Cervical defect, Sclerotic dentin

\section{INTRODUCTION}

The efficacy of dentin bonding systems have been evaluated using intact dentin as a substrate in many studies ${ }^{1-8)}$. From a clinical point of view, however, the dentin adjacent to the caries, the dentin of a cervical defect or the exposed root surface requires resin composite restoration. It has been clarified that the physical and chemical characteristics of this type of dentin is most likely degenerated compared with those of the normal dentin. Fusayama et al. ${ }^{9,10)}$ reported that the caries dentin was classified into two layers, the inner and outer layer, which were either recalcified or not re-calcified physiologically. They invented the caries detector, acid red diluted in propylene glycol, by which the two layers were identified. In addition, the dentin tubules in the inner layer of the caries dentin were filled with debris suggested to be the hardness of this sclerotic dentin. Yoshiyama et al. ${ }^{11)}$ reported that the dentin tubules in the cervical defect indicate a good correlation with the clinical performance of the sensitivity of the tooth and closed dentin tubules did not cause hypersensitivity. These two findings supported the hydro-dynamic theory by Bränström ${ }^{12)}$ who suggested that dentin sensitivity was caused by fluid flow in the dentin tubules. The permeability of the dentin is limited in the sclerotic dentin because the dentin tubules are closed by the debris of the cubic crystals of calcium phosphate. Such a difference in the physical structure between the sclerotic and 
normal dentin possibly affects the efficacy of the dentin bonding system. Ogata et $a l .{ }^{13)}$ measured the micro tensile bond strength to the sclerotic dentin forming a cervical defect; the bond strength was higher in the occlusal dentin wall compared with the apical dentin although both dentin cavity walls exhibited lower bond strength than the normal dentin. Furthermore, Nakajima et al. ${ }^{14)}$ recommended the conditioning of the caries affected dentin using a strong acid such as $40 \%$ phosphoric acid for $15 \mathrm{sec}$ to form a hybrid in the high acid resistance dentin. Many studies concerning dentin bonding materials to the sclerotic dentin, claim that the efficacy of the dentin adhesive deteriorates to the sclerotic dentin because the reduced permeability interrupts hybrid layer formation ${ }^{15)}$.

The primary requirement for the dentin adhesive, however, is to maintain attachment between the unpolymerized resin composite paste and the dentin cavity wall until polymerization of the resin composite is completed. We previously reported that the contraction gap width increased when the functional monomer was omitted from the dentin bonding agent or the dentin cavity wall was decalcified using a strong acid such as $40 \%$ phosphoric acid and the contraction gap was formed between the dentin cavity wall and the resin composite ${ }^{16)}$. Therefore, it is suggested that it is most important to prevent contraction gap formation to maintain a high concentration both of the adhesive monomer and the calcium in the substrate at the adhesive interface and to eliminate the inhibitors of monomer polymerization such as oxygen in the air and water from the dentin tubules ${ }^{17)}$. Thus, separation of the resin composite paste from the dentin cavity wall is prevented and the entire polymerization contraction stress is released from the free surface of the resin composite filling.

The purpose of the present study was to investigate the efficacy of dentin adhesive to sclerotic dentin in a cervical defect.

\section{MATERIALS AND METHODS}

\section{Contraction gap width measurement}

Human incisors or pre-molars with cervical defects which were extracted for periodontal reasons were used. The surface of the defect was ground slightly using a round-shaped steel bur mounted on a low-speed cutting machine. The occlusal or incisal enamel cavity wall was etched with a commercial phosphoric acid gel (Etchant; 3M, MN, USA) (Table 1) for $30 \mathrm{sec}$ and the cavity was rinsed and dried

Table 1 Experimental dentin bonding systems tested

\begin{tabular}{ll}
\hline \multicolumn{1}{c}{ Bonding System } & \multicolumn{1}{c}{ Materials } \\
\hline Enamel etching & Etchant $^{1}$ \\
Dentin conditioner & neutralized $0.5 \mathrm{~mol} / \mathrm{L}$ EDTA $(\mathrm{pH} \mathrm{7.4)}$ \\
Dentin primer & 35 vol\% of glyceryl mono-methacrylate (GM) \\
Dentin bonding agent & Clearfil Photo Bond ${ }^{2}$ \\
\hline${ }^{1} 3 \mathrm{M}$, St Paul, MN, USA, ${ }^{2}$ Kuraray, Osaka, Japan
\end{tabular}


completely. Then an experimental dentin conditioner, $0.5 \mathrm{~mol} / \mathrm{L}$ EDTA neutralized to pH 7.4 was filled in the cavity for $60 \mathrm{sec}$ followed by rinsing and dring. In half of the specimens, the cavity wall was primed with 35 vol\% aqueous solution of glyceryl mono-methacrylate solution $(\mathrm{GM})$ for $60 \mathrm{sec}$ followed by air blasting to eliminate the primer completely, although in half of the specimens the cavity was not primed. A commercial dual-cured bonding agent (Clearfil Photo Bond; Kuraray, Tokyo, Japan) was applied in all the cavities and irradiated for $10 \mathrm{sec}$ followed by a commercial light-activated resin composite (Silux Plus; 3M, MN, USA) filling and irradiated for $40 \mathrm{sec}$. After $10 \mathrm{~min}$ of storing the specimens in water at a room temperature of $24 \pm 1{ }^{\circ} \mathrm{C}$, a section through the center of the cavity of each tooth was prepared on wet carborundum paper. The cavity adaptation of the resin composite was inspected under a microscope and the maximum contraction gap width along the cavity margin was measured using a screw micrometer (Eyepiece Digital; Leitz, Wetzler, Germany) mounted on the ocular lens of a light microscope (Orthoplan; Leitz, Wetzler, Germany).

For the control group, V-shaped cavities with a cavo-surface angle of $135^{\circ}$ were prepared using an experimental diamond point mounted on a high-speed cutting machine in carious-free extracted human incisors or pre-molars as shown in Fig. 1. The V-shaped cavity was restored with the dentin bonding system and resin composite using the same procedures as in the cervical defect. Gap width measurements on the section of the cavity were carried out using the same method as that in the cervical defect and V-shaped cavity, respectively; 40 in total were prepared. The results of wall-to-wall maximum contraction gap measurements were analyzed statistically with Fisher's PLSD test.

\section{Vicker's Hardness measurement}

Micro-Vicker's hardness both of the sclerotic and the normal dentin was determined by measurement at the hardness of the dentin adjacent to the resin cavity margin as presented in Fig. 2. The dentin hardness measurement was performed at five points

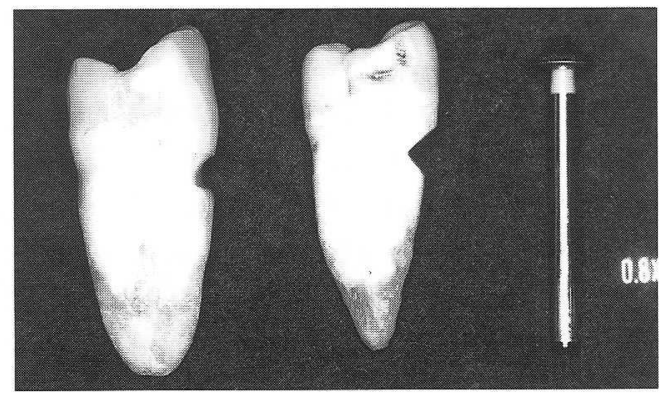

Fig. 1 Cervical defects of a tooth extracted for periodontal reasons, the $\mathrm{V}$-shaped cavity (carvo-surface angle; $135^{\circ}$ ) and an experimental diamond point (Left to right). 


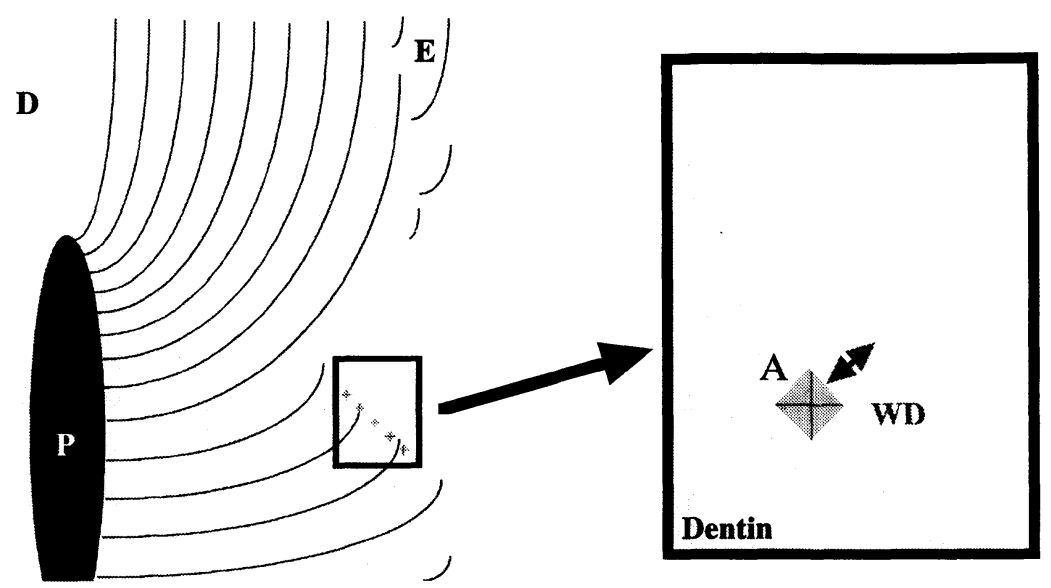

Fig. 2 The micro-Vicker's hardness measurement. (A; Square mark with the micro-Vicker's hardness tester. MA : Measurement area was located less than $3 \mu \mathrm{m}$ from the cavity surface.)

for each specimen using the micro-hardness tester (MVK-E; Akashi, Tokyo, Japan), and the mean value of measurements was employed as the hardness of the specimen. The measurement of normal dentin and the dentin located in the cervical defect was analyzed statistically by Student's t-test.

\section{Scanning Electron Microscopic observation}

The microstructure of normal dentin and the cervical defects were observed with a scanning electron microscope (S-4700; Hitachi, Tokyo, Japan) after critical point drying and spatter coating the specimen with palladium and platinum. For the control group, the specimen in which dentin was etched with the $35 \%$ phosphoric acid gel for $60 \mathrm{sec}$ was observed.

\section{RESULTS}

\section{Maximum contraction gap measurement}

When the dentin cavity wall was primed with GM, complete marginal adaptation was observed in all the specimens prepared regardless of the kind of dentin (Table 2). Without GM priming, cavity adaptation of the resin composite was not observed in all the specimens of the normal dentin whereas complete cavity adaptation was observed in seven specimens out of 10 with a cervical defect. The statistical analysis of difference was insignificant among the three tested groups (Fisher's PLSD test, $\mathrm{p}<0.05)$.

\section{Vicker's Hardness measurement}

Dentin hardness of the dentin located in the cervical defect was significantly decreased compared with that of the dentin located in the artificially prepared V- 
Table 2 Mean and standard deviations of the contraction gap width and Vicker's hardness

\begin{tabular}{cccc}
\hline \multirow{2}{*}{ Bonding Substrates } & \multicolumn{2}{c}{ Contraction gap $(\mu \mathrm{m})$} & \multirow{2}{*}{ Vicker's Hardness } \\
\cline { 2 - 3 } Normal Dentin & $\left.0(10)\right|^{*}$ & $2.34 \pm\left. 0.09(0)\right|^{* *}$ & $59.53 \pm\left. 2.66\right|^{\#}$ \\
Cervical Defect & $0(10)$ & $0.59 \pm\left. 1.18(7)\right|^{*}$ & $49.74 \pm\left. 1.08\right|^{\# \#}$ \\
\hline
\end{tabular}

$\mathrm{N}=10$

Mean \pm SD of the maximum contraction gap width and the number of gap-free cases out of 10 are ( ).

*; Tne values of the contraction gap measurement joined by the line were not significantly different by Fisher's PLSD $(p<0.05)$.

\#; The values of the Vicker's hardness measurement joined by the line were significantly different between two groups by Student's ttest $(p<0.05)$.

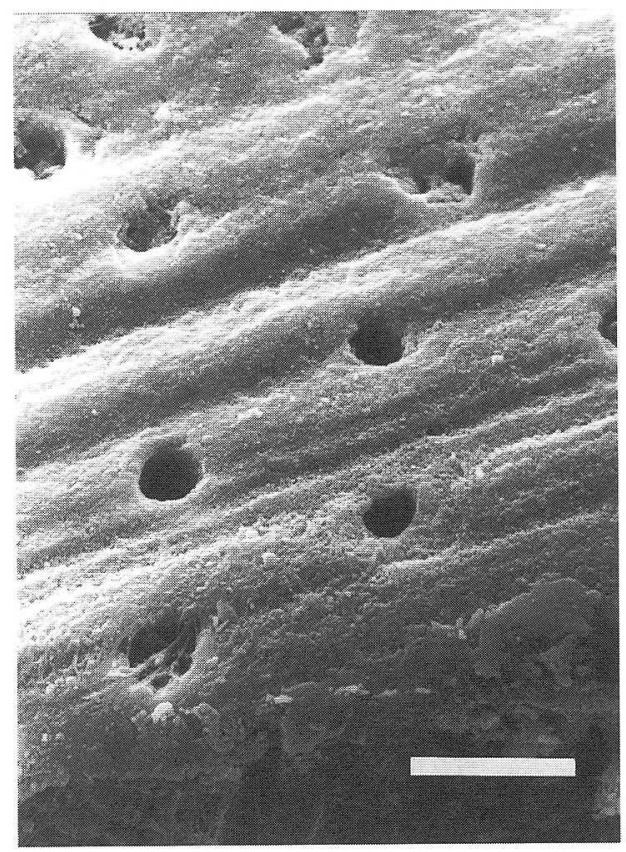

Fig. 3 Scanning electron microscopic (SEM) observation of the normal dentin which was conditioned with $0.5 \mathrm{~mol} / \mathrm{L}$ EDTA for $60 \mathrm{sec}$ $(\times 5,000)$. The bar represents $5 \mu \mathrm{m}$.

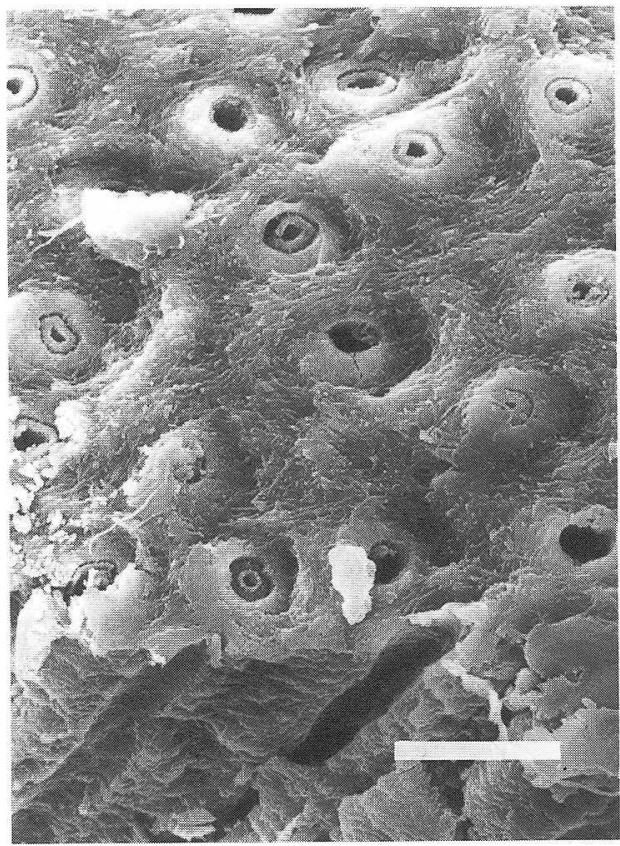

Fig. 4 SEM observation of the sclerotic dentin which was conditioned with $0.5 \mathrm{~mol} / \mathrm{L}$ EDTA for $60 \mathrm{sec}$ $(\times 5,000)$.

shaped cavity (Student's t-test, $\mathrm{p}<0.05$ ).

\section{Scanning Electron Microscopic observation}

In the case of the normal dentin conditioned by EDTA, the smear layer and debris on the dentin surface was completely removed although a dentinal plug and opening of the dentin tubules were observed (Fig. 3). In the case of the sclerotic dentin condi- 


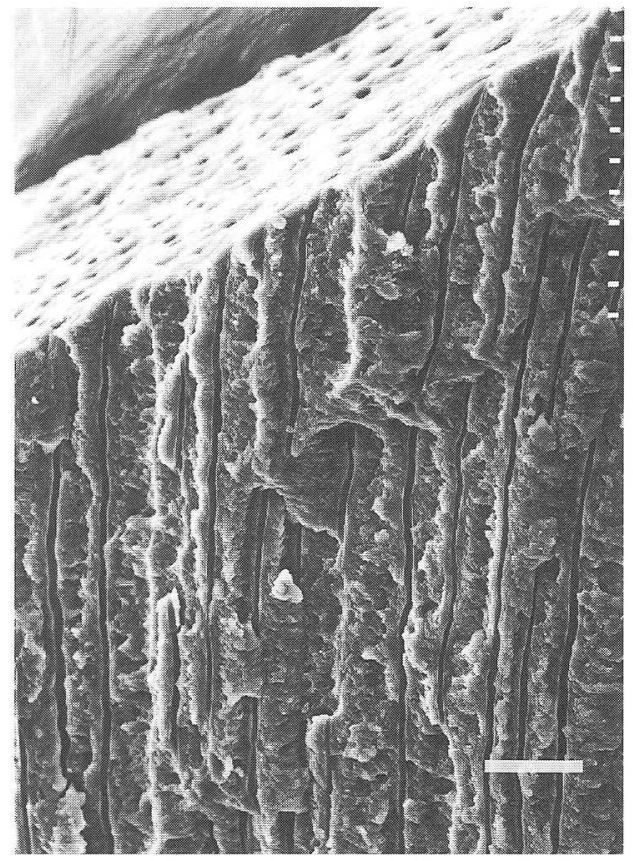

Fig. 5 SEM observation of normal dentin tubules $(\times 500)$. The bar represents $20 \mu \mathrm{m}$.

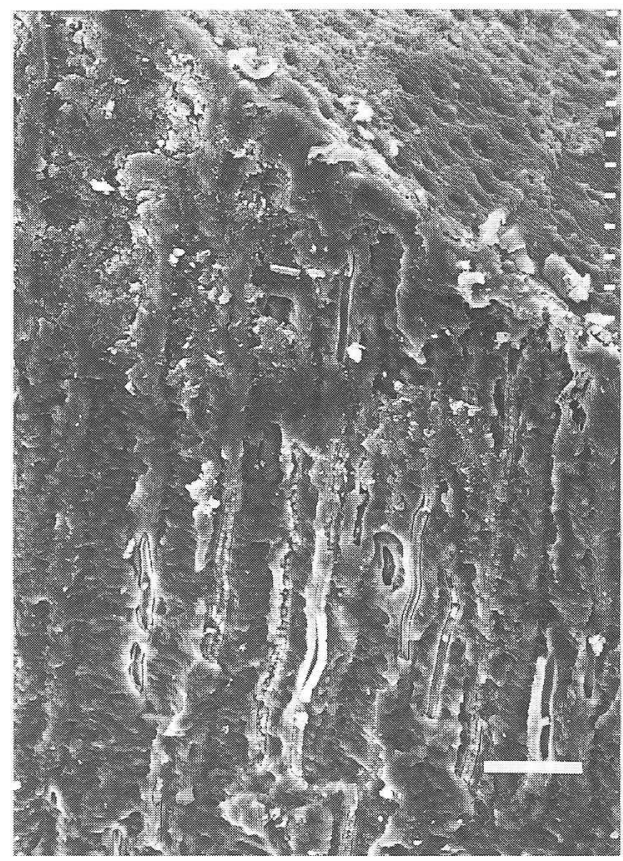

Fig. 7 SEM observation of sclerotic dentin tubules $(\times 500)$. The bar represents $20 \mu \mathrm{m}$.

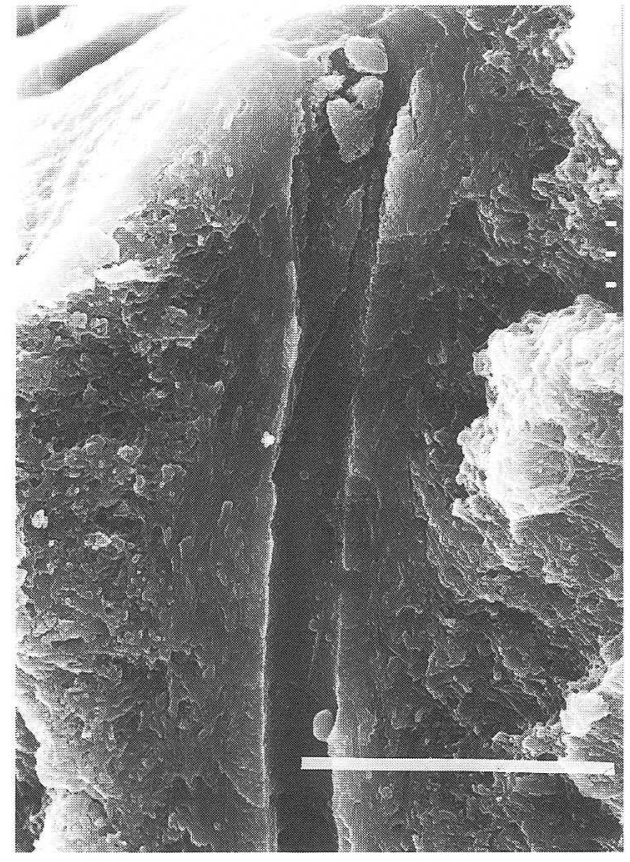

Fig. 6 High magnification of Figure 5 $(\times 5,000)$. The bar represents $5 \mu \mathrm{m}$.

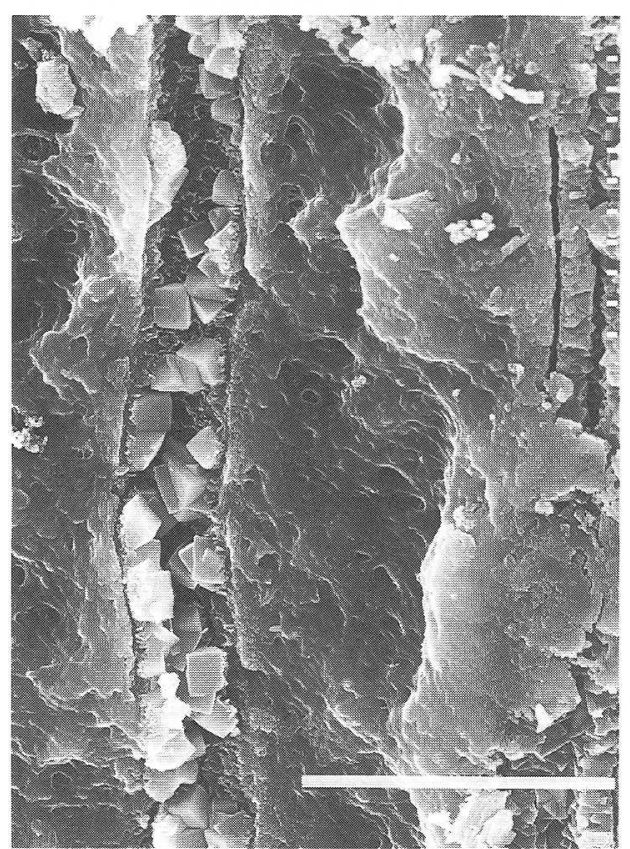

Fig. 8 High magnification of Figure 7 $(\times 5,000)$. The bar represents $5 \mu \mathrm{m}$. 


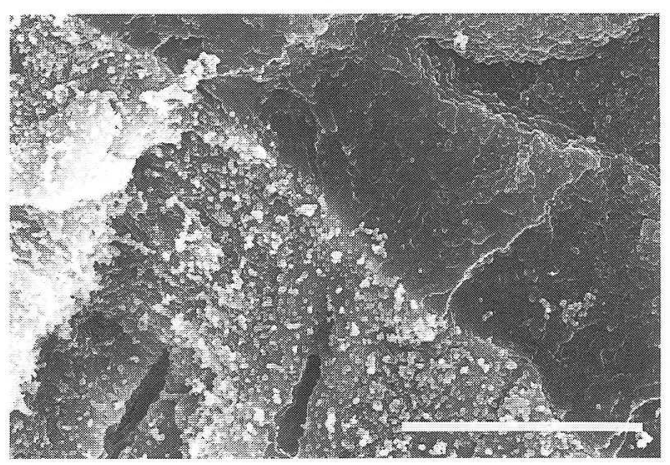

Fig. 9 SEM observation at the adhesive interface of the sclerotic dentin which was cut through in a longitudinal section $(\times 5000)$.

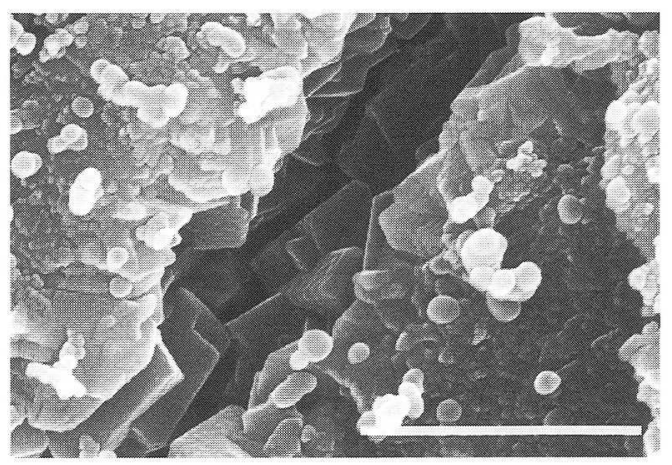

Fig. 10 High magnification of Figure 8 $(\times 30,000)$. The bar represents $1 \mu \mathrm{m}$.

tioned with EDTA, the debris was removed from the dentin surface and opening of the dentin tubules was observed (Fig.4). When the normal dentin was etched with the phosphoric acid, the dentin tubules were opened widely compared with the EDTA conditioning. The intertubular dentin was not decalcified compared with that of the normal dentin. The dentin tubules with cervical defects were closed by crystal-like substances (Figs. 5-10).

\section{DISCUSSION}

The findings in this study were; 1) Complete marginal adaptation was observed in the sclerotic dentin of the cervical defects and intact dentin of the artificially prepared V-shaped cavity when the resin composite was filled into the cavity followed by the experimental dentin bonding system application, which was composed of EDTA conditioning, GM priming, Clearfil Photo Bond application. 2) In seven specimens with cervical defects, the gap formation was prevented even when the GM priming was omitted and cavity adaptation of the resin composite was clearly superior in the cervical defect compared with that in the artificially prepared V-shaped cavity. 3) In the SEM observation, the dentin tubules of a cervical defect were closed by debris with various crystal-like substances. 4) The sclerotic dentin showed an acidresistance compared with the intact dentin, even thougth the Vicker's hardness of the dentin of the cervical defect was slightly reduced.

In the contraction gap measurements, such a discrepancy between the sclerotic and the intact dentin in the un-primed dentin cavity can be explained by the mechanism of GM priming.

As reported by Chigira et $a l .^{18)}$, monomer infiltration through the dentin is considered to be limited by the GM solution. Thus, the concentration of the functional monomer in both the hydrophilic and hydrophobic groups remained high at the adhesive interface. Furthermore, the fluid flow through the dentin tubules was also 
interfered by GM priming; consequently, polymerization of the adhesive monomer was ensured. Such a high monomer concentration at the adhesive interface was detected as a high destiny zone by TEM observations.

As described above, in the contraction gap measurements, such a discrepancy between the sclerotic and the intact dentin in the un-primed dentin cavity can be explained by the mechanism of GM priming.

It was recognized that permeability through the dentin was significantly limited in the sclerotic dentin. Such a reduction of infiltration into the dentin was still limited by GM priming; consequently, the monomer concentration at the adhesive interface was kept sufficiently high to prevent contraction gap formation. For the normal dentin, monomer diffusion into the dentin was not prevented without GM priming and the monomer concentration was too reduced to prevent gap formation. As discussed above, the sclerotic dentin decreased monomer diffusion into the dentin and exhibited an effect such as a dentin priming.

In many studies, however, the mechanism of dentin adhesives is based on hybrid layer formation in the superficial substrate dentin as demonstrated by Nakabayashi ${ }^{19,20)}$. Therefore, acid etching the dentin with a dentin conditioner and monomer infiltration into the collagen-rich dentin layer has been considered essential to obtain bonding between the resin materials and the dentin. Furthermore, the effect of the 2-HEMA based dentin primer has been explained by promoting monomer diffusion into the etched dentin ${ }^{21)}$. We previously reported that the priming effect of the 2-HEMA primer was claerly inferior to that of the GM primer probably because the 2-HEMA primer increased permeability of the monomer into the dentin and decreased the adhesive monomer concentration at the adhesive interface ${ }^{18)}$.

In 1991, the mechanism of the dentin primer was explained by expanding the microspace among the dentin collagen network which was collapsed by dentin conditioning ${ }^{22)}$. Subsequently, the adhesive monomer easily penetrated into the expanded microspace and formed the hybrid layer. In these studies, however, the efficacy of the dentin bonding system was estimated by measuring the tensile bond strength to the flat dentin surface. In addition, the fracture inside the resin composite or the dentin substrate was frequently observed even when the specimen was dumb-bell shaped to fracture at the adhesive interface. It is noteworthy that the clinical contraction gap was formed between the dentin cavity wall and the resin composite paste and never inside the resin composite or inside the dentin along the cavity margin. This clinical phenomenon suggested the conflict of estimating the efficacy of the dentin adhesive by the bond strength measurement. Hasegawa et al. ${ }^{23)}$ claimed that the mechanical properties of the resin composite significantly affected the bond strength of the dentin bonding systems because the higher tensile bond strength was measured by the resin composite that exhibited the higher tensile strength. It was consistent that the resin composite with higher mechanical properties did not always exhibit improved adaptation in the dentin cavity. It is noteworthy that the efficacy of the dentin bonding system did not deteriorate in the cervical defect even though the hardness of the dentin of the cervical defect was slightly reduced. We previously 
reported that decalcification or the reduction of hardness on the dentin cavity wall increased the contraction gap width ${ }^{24)}$. The results in the present study suggested that the closed dentin tubules or the limited permeability in the sclerotic dentin played a more important role for the establishment of marginal adaptation of the resin composite than the slight reduction in dentin hardness. We previously demonstrated that the dentin hardness was decreased by only $15 \%$ by the EDTA conditioning $^{24)}$. It is easily suggested that conditioning with a strong acid such as $40 \%$ phosphoric acid possibly deteriorated the marginal integrity of the resin composite in the sclerotic dentin cavity.

As discussed above, the dentin tubules of a cervical defect were closed by debris with various crystal-like substances. Such substanses in the dentin tubles of the sclerotic dentin not only improved the resistance to acid, but the fluid flow through the dentin tubles from deep inside was also affected, and decreased the momoner diffusion into the dentin. Consequently, the monomer concentration at the adhesive interface was kept sufficiently high to prevent contraction gap formation. These effects occurred after the monomer had infiltrated through the dentin and are considered to be limited by the GM priming.

The efficacy of the experimental dentin bonding system to the sclerotic dentin was superior to that to the normal dentin especially when the dentin cavity was not primed with a GM solution. It was suggested that such improved bonding efficacy was based on the limited permeability of the sclerotic dentin which causes a high adhesive monomer concentration on the adhesive interface. Clinically, it is recommended that the sclerotic dentin in a cervical defect be conditioned with a mild conditioner such as EDTA and conservatively restored with a resin composite.

\section{CONCLUSIONS}

The sclerotic dentin in a cervical defect and root dentin is more suitable as a substrate for a dentin adhesive than the intact dentin. The sclerotic dentin shows effects such as a priming, and cavity adaptation of the resin composite does not deteriorate even when the cavity is not primed with a GM solution.

\section{REFERENCES}

1) Asmussen, E.: Composite restorative resins. Composition versus wall-to-wall polymerization contraction, Acta Odontol Scand $33: 337-344,1975$.

2) Brännström, M, Nordenvall, K. J. and Glantz, P. O.: The effect of EDTA-containing surface-active solutions on the morphology of prepared dentin - An in vitro study,$- J$ Dent Res 59(7): 1127-1131, 1980.

3) Bowen, R. L., Cobb, E. N. and Rapson, J. E.: Adhesion bonding of various materials to hard tooth tissues: Improvement in bond strength, $J$ Dent Res 61 : 1070, 1982.

4) Munksgaard, E. C. and Asmussen, E.: Bond strength between dentin and restorative resins mediated by mixtures of HEMA and glutaraldehyde, J Dent Res 63(8): 1087-1089, 1984.

5) Itoh, K., Hashimoto, K. and Wakumoto, S.: Effect of GLUMA concentration on adhesion to dentin, Japan J Conserv Dent 28(3):895-901, 1985. (in Japanese) 
6) Chigira, H., Itoh, K. and Wakumoto, S.: Marginal adaptation of nine commercial intermediate resins, Dent Mater $7: 103-106,1991$.

7) Manabe, A., Itoh, K. and Wakumoto, S.: The optimum combination of dentin bonding systems, Dent Mater $7: 145-147,1991$.

8) Chigira, H., Manabe, A., Hasegawa, T., Yukitani, W., Fujimitsu, T., Itoh, K., Hisamitsu, H. and Wakumoto, S.: Efficacy of various commercial dentin bonding systems, Dent Mater $10: 363-368,1994$.

9) Fusayama, T. and Terashima, S.: Differentiation of two layers of carious dentin by staining, Bull Tokyo Med Dent Univ 19(1) : 83-92, 1972.

10) Fusayama, T.: Two layers of carious dentin; diagnosis and treatment, Oper Dent 4(2): 63-70, 1979.

11) Yoshiyama, M., Suge, T., Kawasaki, A. and Ebisu, S.: Morphological characterization of tube-like structures in hypersensitive human radicular dentine, $J$ Dent 24(1-2) : 57-63, 1996.

12) Brännström, M., Linden, L. A. and Astrom, A.: The hydrodynamics of the dental tubule and of pulp fluid. - A discussion of its significance in relation to dentinal sensitivity-, Caries Res 1(4): 310-7, 1967.

13) Ogata, M., Nakajima, M., Sano, H. and Tagami, J.: Effect of dentin primer application on regional bond strength to cervical wedge-shaped cavity walls, Oper Dent 24(2):81-8, 1999.

14) Nakajima, M., Sano, H., Burrow, M.F., Tagami, J., Yoshiyama, M., Ebisu, S., Ciucchi, B., Russell, C. M. and Pashley, D. H.: Tensile bond strength and SEM evaluation of caries-affected dentin using dentin adhesives, J Dent Res 74(10): 1679-88, 1995.

15) Yoshiyama, M., Sano, H., Ebisu, S., Tagami, J., Ciucchi, B., Carvalho, R. M. Johnson, M. H. and Pashley, D. H.: Regional strengths of bonding agents to cervical sclerotic root dentin, J Dent Res 75(6) : 1404-13, 1996.

16) Manabe, A., Itoh, K., Tani, C., Hisamitsu, H. and Wakumoto, S.: Effect of the functional monomers in the dentine bonding agents, Dent Mater $J$ 18(1): 116-123, 1999.

17) Sano, H., Yamashita, T., Itoh, K., Wakumoto, S. and Hisamitsu, H.: Effect of decalcification of dentin on the efficacy of dentin adhesive - Cleansing time with 10-3 solution vs contraction gap width of resin composite in dentin cavity -, Journal of Japanese Society for Dental Materials and Devices Special Issue 28(15) : 162-163, 1996. (in Japanese)

18) Chigira, H., Itoh, K., Tachikawa, T., Wakumoto, S. and Hisamitsu, H.: Bonding efficacy and interfacial microstructure between resin and dentine primed with glyceryl methacrylate, J Dent 26 : 157-163, 1998

19) Nakabayashi, N.: Resin reinforced dentin due to infiltration of monomers into the dentin at the adhesive interface, $J J$ Dent Mater $1: 78-81,1982$. (in Japanese)

20) Nakabayashi, N., Kojima, K. and Masuhara, E.: The promotion of adhesion by the infiltration of monomers of into tooth substrates, $J$ Biomed Mater Res 16 : 265-273, 1982.

21) Nakabayashi N and Takarada K: Effect of HEMA on bonding to dentin, Dent Mater 8 : 125-130, 1992.

22) Sugizaki, J.: The effect of the various primers on the dentin adhesion of resin composites -SEM and TEM observation of the resin impregnated layer and adhesion promoting effect of the primers-, Japan $J$ Conserv Dent 34(1) : 228-265, 1991. (in Japanese)

23) Hasegawa, T., Itoh, K., Yukitani, W., Koike, T., Hisamitsu, H. and Wakumoto, S.: Effect of mechanical properties of resin composites on the efficacy of dentin bonding system, Operative Dentistry $24: 323-330,1999$.

24) Chiba, M., Itoh, K. and Wakumoto, S.: Effect of dentin cleansers on the bonding efficacy of dentin adhesive, Dent Mater J 8:76-85, 1989. 